\title{
Engorda del bagre de canal y la tilapia con piensos de harina de jícaro
}

Roberto Alexander Martínez Lagos ${ }^{1}$

Oscar Colindres Varela ${ }^{2}$

Marilena Rodríguez Velasquez ${ }^{3}$

Kaina Alvarado ${ }^{4}$

\section{RESUMEN}

En la zona sur de Honduras se ha visualizado a la acuicultura, como una alternativa para la obtención de proteína de alta calidad parala población rural de bajos ingresos, en esta zona la obtención de insumos locales alternativos de bajo costo y especies acuícolas de rápido crecimiento es clave para asegurar el éxito de los procesos que afiancen la seguridad alimentaria en la población. Una opción para alcanzar este objetivo es el uso del bagre de canal (Ictalurus punctatus) como especie de trabajo y la harina de jícaro (Crescentia alata) como base para la elaboración de alimento de bajo costo. En este estudio se evaluó el crecimiento del bagre de canal y la tilapia en fase de engorda obteniendo un factor de conversión de 3.56:1 y 4.19: 1 respectivamente, con alimento con un contenido de $25 \%$ de proteína cruda y $70 \%$ de su composición a base de harina de jícaro. La tilapia obtuvo un incremento de 0.68 gramos de peso diario y el bagre 2.76 gramos; el factor de condición en las dos especies al final del experimento fue apropiado.

Palabras clave: Ictalurus punctatus, Tilapia, Crescentia alata.

\footnotetext{
${ }^{1}$ Profesor de la carrera de Ingeniería Acuícola, Centro Universitario Regional de Litoral Pacífico (CURLP), UNAH: robertomartinez@unah.edu.hn

${ }^{2}$ Profesor del departamento académico de ciencias Centro Universitario Regional de Litoral Pacífico (CURLP), UNAH.

3 Profesor del departamento químico, biológico y de la salud, Centro Universitario Regional de Litoral Pacífico (CURLP), UNAH

${ }^{4}$ Estudiante de la carrera de Ingeniería Acuícola, Centro Universitario Regional de Litoral Pacífico (CURLP), UNAH
} 


\section{ABSTRACT}

In the southern region of Honduras it has been viewed aquaculture as an alternative for obtaining high quality protein for low-income rural population. In this area obtaining alternative low-cost local inputs and fast-growing aquaculture is key to ensuring the success of the processes that strengthen food security in the population. One option for achieving this goal is the use of channel catfish (Ictalurus punctatus) as specie of culture and flour Jícaro (Crescentia alata) as a basis for the development of low-cost food. In this study the growth of channel catfish and tilapia in fattening stage was evaluated obtaining a conversion factor of 3.56: 1 and 4.19: 1 respectively, with feed containing $25 \%$ crude protein and $70 \%$ of its composition flour-based Jícaro. The tilapia obtained an increase in daily weight 0.68 grams and 2.76 grams the catfish; the condition factor in the two species at the end of the experiment was appropriate.

Keywords:Ictaluruspunctatus, Tilapia, Crescentiaalata 


\section{INTRODUCCIÓN}

El pescado es la principal fuente de proteína de origen animal en los países en desarrollo, según Moffitt \& Cajas-Cano (2014) afirman que el pescado representó en el 2010 el $17 \%$ del aporte de la proteína animal a nivel mundial como alimento para las personas y el $6.5 \%$ de todas las proteínas consumidas. Además se estima que más de 158 millones de personas en el mundo dependen directamente de las actividades pesqueras (pesca, cría de peces, elaboración y comercio); más del $90 \%$ de estas personas son operadores a pequeña escala que viven en países en desarrollo (HLPE, 2014).

En Honduras el cultivo del camarón blanco (Litopenaeus vannamei) y la tilapia (Oreochromis sp.) son las actividades acuícolas que mayor aporte tienen a la economía del país, aunque el cultivo de peces se inicia como una actividad de seguridad alimentaria en la zona rural desde 1960 (Costa-Pierce y Rakocy, 1997) y se ha desarrollado en la actualidad como uno de los principales rubros de exportación de la nación; por esta razón existe el interés por la diversificación acuícola. Con esta visión la industria cataloga estos estudios como una actividad de previsión por los problemas que los monocultivos suelen presentar y para aprovechar el potencial de la biodiversidad presente en el país; con estos fines, son de especial interés las especies resistentes en acuicultura que se puedan cultivar en estanques rústicos de tierra, dado que el gobierno central desea desarrollar de forma masiva infraestructura de cosecha de agua, con doble propósito que sirva además para cultivar peces (SAG, 2016).El bagre de canal es una especie de gran importancia en acuicultura en Norte América y Asia, por lo cual existe la información necesaria $(F A O, 2014)$ que facilitaría su cultivo en Honduras, además esta especie se encuentra en estado silvestre en el país en la cuenca del Río Ulúa, al proponerla como una alternativa de seguridad alimentaria para la zona rural del país y además la utilización de alimentos alternativos de bajo costo para la engorda es una opción en este propósito, planteada por diversos autores (Poot-Lopez, G., Gasca-Leyva, E., Olvera-Novoa, M. 2012; Okeyo y Montgomery,1992; Hernández-Valencia, 2014) para alcanzar este objetivo.

En el presente estudio se evaluó el desempeño acuícola del bagre de canal engordado con piensos elaborados artesanalmente a base de harina de jícaro, como alimento alternativo de bajo costo y se comparó en cultivo con la tilapia con el objetivo de proponer la especie más apropiada para la acuicultura de pequeña escala o subsistencia, reforzando la seguridad alimentaria en el sector rural de la zona sur del país. 


\section{MÉTODOS}

El enfoque de este estudio es de tipo cuantitativo, con un diseño experimental controlado, para lo cual se llevó acabo la siguiente metodología:

\section{a. Colecta de organismos}

Los peces de las dos especies de trabajo se colectaron en el valle de Comayagua, las tilapias de la variedad Red Florida fueron donadas por la Estación Acuícola Experimental El Carao, los bagres fueron capturados en las instalaciones de la Finca Acuícola Los Palillos, ambos grupos de peces fueron transportados en un vehículo pick up al Centro Universitario Regional del Litoral Pacífico (CURLP) en un tanque de 750 litros de agua y con oxígeno de soporte.

\section{b. Mantenimiento de los organismos}

La cantidad de organismos seleccionada se ajustó al número de bagres silvestres capturados, de acuerdo a esto se sembraron 6 peces a una densidad de 1.2 peces por metro cubico, en cada una de las unidades experimentales los organismos se mantuvieron en dos tanques de membrana de polietileno de 5 toneladas y se alimentaron 1 vez al día calculando el $3 \%$ de la biomasa establecida (FAO, 1988) y reajustando la ración conforme se realizaron las biometrías a lo largo del experimento cada 18 días. Se realizó un recambio parcial del $10 \%$ del volumen total de agua del sistema una vez a la semana; los parámetros de calidad de agua del sistema se monitorearon 2 veces a la semana verificando, amonio, fosfatos, nitritos, $\mathrm{pH}$, oxígeno y temperatura; para este fin se utilizóun kit colorimétrico de la marca MarsFishcare y un oxigenómetro portátil (YSI 550A).

\section{c. Preparación del alimento artesanal}

La preparación del alimento se realizó utilizando como insumos harina de jícaro secada al sol con un contenido de proteína cruda (PC) del $26 \%$, harina de cabeza de camarón comercial de la fábrica Prohalca con un contenido (PC) $51 \%$ y harina de trigo comercial marca Gold Star(PC) 8\%. Se utilizó el método de tanteo para el balanceo del alimento, obteniendo un pienso con un $25 \%$ de PC, con una composición del $70 \%$ de harina de jícaro, $20 \%$ harina de trigo y $10 \%$ harina de cabeza de camarón, para el proceso de peletización se utilizo un molino manual de hierro marca Corona. 


\section{d. Biometrías de los peces}

Para monitorear el crecimiento de los peces se realizaron 6 biometrías en el transcurso del experimento, utilizando un ictiómetro y el peso se evaluó utilizando una balanza mecánica de triple brazo marca Ohaus.

\section{Análisis estadístico}

Se realizó un análisis del factor de condición simple como indicador de calidad para conocer el grado de robustez de los peces (Le Cren, 1951., Rodríguez - Gutiérrez, 1992). El factor de condición simple se calculó mediante la siguiente fórmula $=$ $\left(\mathrm{W} / \mathrm{LP}^{\mathrm{b}}\right.$ ) en donde $\mathrm{K}=$ factor de condición simple, $\mathrm{W}=$ peso en gramos $\mathrm{LP}=\mathrm{Longitud}$ total en centímetros y $b=a$ la pendiente de la correlación $W$ contra LP.

Para evaluar el factor de condición en los organismos al finalizar el experimento se utilizó la prueba de U de Mann-Whitney, utilizando el programa Statistica versión 5.5. Se calculo el factor de conversión alimenticia de acuerdo al cálculo de la proporción existente entre la cantidad de alimentos distribuidos (en $\mathrm{kg}$ ) y la ganancia de peso de los peces (en $\mathrm{kg}$ ), en el mismo período de tiempo.

\section{RESULTADOS}

En el desarrollo del experimento se monitorearon los parámetros básicos de calidad de aguas que se presentan

\section{Tabla 1. Parámetros monitoreados en el sistema de cultivo}

\begin{tabular}{|l|l|l|l|l|l|}
\hline Temperatura ${ }^{\circ} \mathrm{C}$ & Oxígeno \% & Amonio & Fosfatos & $\mathrm{pH}$ & Nitritos \\
\hline $30 \pm 1.61$ & $81 \pm 20$ & $1.2 \pm 1$ & $0,28 \pm 0.006$ & $7.78 \pm 2.41$ & $0.02 \pm 0.01$ \\
\hline
\end{tabular}

Fuente: elaboración propia.

En el cultivo de tilapias se obtuvo una ganancia en peso de 61.6 gramos de peso por organismo en los 89 días que duro el experimento, con un incremento diario de 0.68 gramos lo que representa una tasa de crecimiento diario de 1.1\%; en los bagres se obtuvo una ganancia en peso de 246 gramos de peso por organismo, el incremento 
diario fue de 2.76 gramos lo que representa el 1.12\%. El factor de conversión que presentaron las tilapias fue de 4.19:1 y el de los bagres fue de 3.56:1 el comportamiento en el cultivo en ambas especies se presenta en el gráfico 1.

Gráfico 1. Engorde de tilapia y bagre de canal alimentados con piensos a base de harina de jícaro.

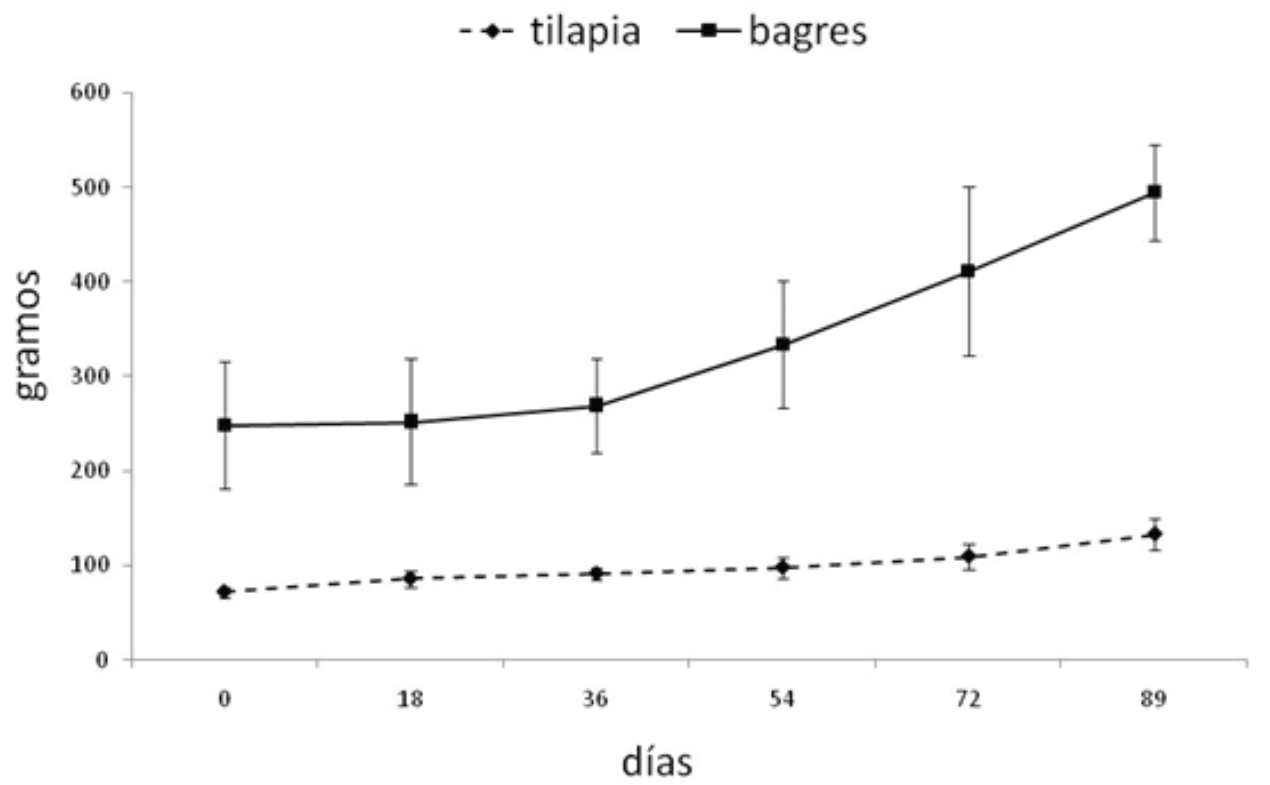

Fuente: elaboración propia.

El análisis del factor de condición simple (F.C.S.) donde R representa el coeficiente de correlación de las variables de estudio, R2 el ajuste del modelo lineal en este caso, $P$ el valor del estadístico calculado y $\sigma$ la desviación estándar. Las demás variables se definieron con anterioridad, se presenta en la tabla 2.

Tabla 2. Factor de condición simple en tilapias y bagres al final del experimento

\begin{tabular}{|c|c|c|c|c|c|c|c|}
\hline Organismos & $\mathrm{N}^{\circ}$ organismos & b & $\sigma$ & $\mathbf{R}^{2}$ & $\mathbf{R}$ & $\mathbf{P}$ & F.C.S. \\
\hline tilapias & 6 & 2.96 & 0.001 & 0.89 & 0.94 & \multirow[t]{2}{*}{$<0.05$} & 1.81 \\
\hline bagres & 6 & 2.95 & 0.08 & 0.80 & 0.9 & & 1.08 \\
\hline
\end{tabular}




\section{DISCUSIÓN}

En el desarrollo del experimento, el sistema utilizado trata de simular las condiciones de cultivo de un sistema extensivo con densidades de siembra bajas y un recambio del 10\% semanal nos permitió mantener los peces dentro de los rangos establecidos de los parámetros evaluados en el cultivo del bagre (Morris, 1993; Wyatt, T., Barkoh, A., Martínez, J., y Sparrow R. 2006) y la tilapia (Saavedra, M. 2003., DeLong D. P., Losordo, T. M., y Rakocy J. E. 2009) lo que se confirmóal tener una mortalidad de 0\% en los organismos a lo largo del experimento. En las dos especies evaluadas se inició el experimento en organismos con peso en fase de engorde aunque de diferentes tallas ya que los bagres fueron colectados estado silvestre; el desempeño de las tilapias en el estudio se considera pobre en $1.1 \%$ diario de ganancia en peso por debajo de los estándares establecidos para cultivos industriales cuyo rango se establece entre el 3.2 al 1.8\%pero dentro de los estándares de cultivos extensivos de la especie. En el bagre el desempeño de 1.12\% diario de ganancia en peso también se considera por debajo de los estándares de los cultivos industriales cuyo rango se establece entre 1.3 al 1.7\% (Ceballos-Orosco y Velásquez-Escobar, 1988) pero dentro de los rangos obtenidos en cultivos artesanales o policultivos de la especie (Navarro-Hurtado, 2002);al evaluar la ganancia en peso en el desarrollo del experimento fue mayor en el bagre de canal que en la tilapia. Tomando en cuenta las condiciones de cultivo en el desarrollo del experimento se deduce que en condiciones de estanques rústicos donde la productividad primaria zooplancton, microorganismos autótrofos y heterótrofos, macro algas y organismos bentónicos pueden dar aportes significativos en la nutrición y crecimiento de los peces (Martínez-Córdova,et al., 2010) las especies evaluadas alcancen mejores crecimientos.

El factor de conversión de las especies evaluadas en el desarrollo del experimento no fue satisfactorio en comparación con los valores recomendados en cultivos industriales, en tilapia el rango de valores recomendable es de 1.4 a 2.5: 1 y en bagre de 1.6 a 3:1 (Ceballos-Orosco y Velásquez-Escobar, 1988) mostrando un mejor desempeño el bagre con valores más cercanos a los recomendados en cultivos a escala industrial; estos resultados pueden justificarse por la calidad del alimento utilizado en el experimento, el cual se elaboró de forma artesanal con semillas de jícaro crudas y es posible que existan deficiencias en cuanto a los requerimientos nutricionales de las especies evaluadas en relación al alimento proporcionado o elementos anti nutricionales desconocidos en el mismo que puedan afectar el crecimiento de los peces. Se propuso el uso de la harina de jícaro como ingrediente principal en el desarrollo de la dieta, dado que su perfil de nutrientes básicos es atractivo (Figueroa-Madrid y Bressani, 2000), la capacidad de la tilapia y el bagre de canal para aprovechar alimentos 
de origen vegetal (Diana, Lin y Jaiyen, 1997; Olvera-Novoa, Domínguez-Cen, Olivera-Castillo y Martínez-Palacios,1998; Tuker, 2003), la abundancia de la materia prima en la zona sur de Honduras y su bajo costo.

A pesar de no alcanzar los pesos sugeridos en el cultivo, las dos especies presentaron un factor de condición apropiado aunque se observaron diferencias significativas entre ellas, lo que nos indica el estado de bienestar y robustez de los peces (Rodríguez-Gutiérrez, 1992), las tilapias presentaron un mayor factor de condición, posiblemente la correspondencia se deba al alto contenido de lípidos presentes en la semilla de jícaro el cual es $38 \%$ en base seca, lo que favorece la acumulación de grasa en los peces, no por ello una mayor formación de musculo que genere un mayor peso en carne.

\section{CONCLUSIONES}

De acuerdo con los resultados obtenidos, el bagre de canal se adapta bien y presenta un mejor desempeño en engorde que la tilapia en las condiciones ambientales de la zona sur de Honduras. La harina de jícaro puede ser un alimento complementario en el cultivo de bagre o tilapia en la zona sur de Honduras, pudiendo disminuir los costos de producción por la compra de alimento balanceado.

\section{AGRADECIMIENTOS}

Se agradece a la DICyP de la UNAH por la beca proporcionada para el desarrollo de este estudio.

\section{BIBLIOGRAFÍA}

Ceballos-Orozco, L., Velázquez-Escobar, M. (1988). Perfiles de la alimentación de peces y crustáceos en los centros y unidades de producción acuícola en México. México: Secretaria de Pesca Dirección General de Acuacultura. Recuperado de http://www.fao.org/docrep/field/003/ab460s/AB460S00.htm\#TOC 
Costa-Pierce, A. \& Rakocy, J. E. (Eds.). (1997). Tilapia Aquaculture in the Americas. World Aquaculture Society. Vol. 1, $258 \mathrm{p}$.

DeLong D. P., Losordo, T. M., Rakocy J. E. (2009). Tank Culture of Tilapia. USA: Southern Regional Aquaculture Center, publication $\mathrm{N}^{\circ} 282$.

Diana, J.S., Lin, C.K. y Jaiyen, K. (1994). Supplemental feeding of tilapia in fertilized ponds. Journal of World Aquaculture. Soc., 25(4): 497-506.

FAO. (1988). Perfiles de la alimentación de peces y crustáceos en los centros y unidades de producción acuícola en México. Pachuca, Hidalgo: Organización de las Naciones Unidas para la Agricultura y la Alimentación.

Moffitt, C. M., \& Cajas-Cano, L. (2014). Blue growth: the 2014 FAO state of world fisheries and aquaculture. Fisheries, 39(11), 552-553.

FAO. (2014). Programa de información de especies acuáticas, Ictalurus punctatus (Rafinesque, 1818). Recuperado de http://www.fao.org/fishery/culturedspecies/Ictalurus_punctatus/es

Figueroa-Madrid, S. A. y Bressani, R. (2000). Recursos alimenticios vegetales con potencial de explotación agroindustrial de Guatemala: Elaboración de leche vegetal a partir de la semilla del fruto de morro (Crescentia alata). Archivos Latinoamericanos de Nutrición, 50(2), 164-170. Recuperado de http://www.scielo.org.ve/scielo.php

Hernández-Valencia, J.,Jiménez, M., Montejo, Z., Guillermo, Carrillo, L. (2014). Manual: Elaboración de alimento alternativo para la producción de Tilapia. México: Instituto Tecnológico de Salina Cruz, Departamento de Acuicultura.

HLPE (2014). La pesca y la acuicultura sostenibles para la seguridad alimentaria y la nutrición. Un informe del Grupo de alto nivel de expertos en seguridad alimentaria y nutrición del Comité de Seguridad Alimentaria Mundial. FAO. Roma. 133 p.

Le Cren, E. D. (1951). The length-weight relationship and seasonal cycle in gonad weight and condition in the perch (Perca fluviatilis). Journal of Animal Ecology. 20(2): 201-219.

Martínez, L. R., Martínez, M., López, A., Miranda, A., Ballester, E. y Porchas, M. A. (2010). Alimento Natural en Acuacultura: una revisión actualizada. En: Cruz-Suarez, L.E., Ricque-Marie, D., Tapia-Salazar, M., Nieto-López, M.G., Villarreal-Cavazos, D. A., Gamboa-Delgado, J. (Eds), Avances en Nutrición Acuícola X Memorias del X Simposio Internacional de Nutrición Acuícola. 8-10 de noviembre, San Nicolás de los Garza, N. L., México: Universidad Autónoma de Nuevo León.

Morris, J.E. (1993). Pond Culture of Channel Catfish in the North Central Region. Extension fisheries/aquaculture specialist, Department of Animal Ecology.Lowa:Lowa State University. 
Navarro-Hurtado A. (2002). Ensayo de dos modelos de policultivo empleando bagre de canal (Ictalurus punctatus) tilapia hibrida (Oreochromis niloticus vs Oreochromis mossabicus) y langostino (Macrobachium tenellum) en estanques semi rústicos caso Jocotepec, Jalisco. (Tesis de maestría). Universidad de Colima México, México.

Okeyo, D., y Montgomery, W. (1992). Ingestion, growth and conversion efficiency in the Blue Tilapia, Oreochromis aureus, when fed on three aquatic macrophytes. Journal of the Arizona-Nevada Academy of Science. Vol. (24/25): 1-10.

Olvera-Novoa, M.A., L.J. Domínguez-Cen, L. Olivera-Castillo., y C.A. Martínez-Palacios. (1998). Effect of the use of the microalgae Spirulina maxima as fish meal replacement in diets for tilapia, Oreochromis mossambicus(Peters), fry. Aquacult., Res., 29 (10): 709-715.

Poot-Lopez,G., Gasca-Leyva, E. y Olvera-Novoa, M. (2012). Producción de tilapia nilótica (Oreochromis niloticus L.) utilizando hojas de chaya (Cnidoscolus chayamansa McVaugh) como sustituto parcial del alimento balanceado. Lat. Am. J. Aquat. Res., 40(4): 835-846.

Rodríguez - Gutiérrez, M. (1992). Técnicas de evaluación cuantitativa de la madurez gonádica en peces. México: Editorial AGT,

Saavedra, M. A. (2003). Introducción al Cultivo de Tilapia. Coordinación de Acuicultura, Departamento de Ciencias Ambientales y Agrarias, Facultad de Ciencia, Tecnología y Ambiente. Managua: Universidad Centroamericana.

SAG. (2016). Inicia construcción masiva de cosechas de agua. Recuperado de http://www.sag.gob.hn/sala-de-prensa/noticias/ano-2016

Tucker, C. (2003). Channel catfish. In Lucas, J.S. \& Southgate, P.C. (eds.), Aquaculture: Farming Aquatic Animals and Plants, pp. 346-363. John Wiley \& Sons.

Wyatt, T., Barkoh, A., Martinez, J., Sparrow R. (2006). Guidelines for the culture of blue and channel catfish. Management data series. Texas: Texas parks and wildlife department in land fisheries division. 